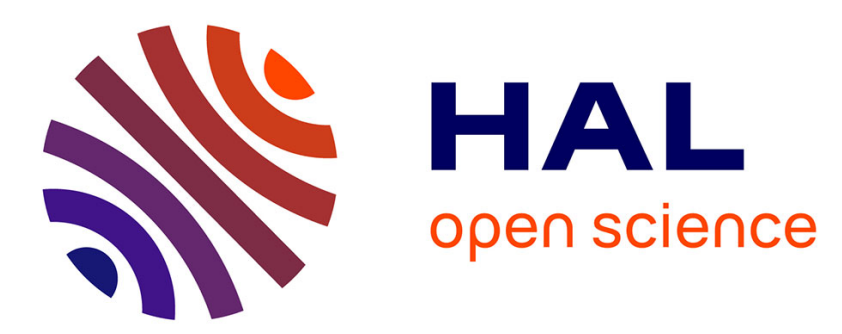

\title{
Axial fatigue of a gas-nitrided quenched and tempered AISI 4140 steel: effect of nitriding depth
}

Nathalie Limodin, Yves Verreman, T. Tarfa

\section{To cite this version:}

Nathalie Limodin, Yves Verreman, T. Tarfa. Axial fatigue of a gas-nitrided quenched and tempered AISI 4140 steel: effect of nitriding depth. Fatigue and Fracture of Engineering Materials and Structures, 2003, 26 (9), pp.811-820. 10.1046/j.1460-2695.2003.00682.x . hal-03325226

\section{HAL Id: hal-03325226 \\ https://hal.science/hal-03325226}

Submitted on 24 Aug 2021

HAL is a multi-disciplinary open access archive for the deposit and dissemination of scientific research documents, whether they are published or not. The documents may come from teaching and research institutions in France or abroad, or from public or private research centers.
L'archive ouverte pluridisciplinaire HAL, est destinée au dépôt et à la diffusion de documents scientifiques de niveau recherche, publiés ou non, émanant des établissements d'enseignement et de recherche français ou étrangers, des laboratoires publics ou privés. 
This document is a personal copy of the accepted version of the paper:

Limodin, N., Y. Verreman, et T. N. Tarfa. «Axial fatigue of a gas-nitrided quenched and tempered AISI 4140 steel: effect of nitriding depth ». Fatigue AND Fracture of Engineering Materials and Structures 26 (2003): 811-20.

The final publication is available at:

https://onlinelibrary.wiley.com/doi/abs/10.1046/j.1460-2695.2003.00682.x

\title{
Axial fatigue of a gas nitrided quenched and tempered AISI 4140 steel: Effect of nitriding depth
}

\author{
N. LIMODIN*, Y. VERREMAN* and T. N. TARFA** \\ *Department of Mechanical Engineering, Ecole Polytechnique de Montréal, Case Postale 6079, \\ Succursale Centre-ville, Montréal H3C 3A7, Québec, Canada and **Technology Department, Nitrex \\ Metal Inc., 3474 Poirier bd, Saint-Laurent H4R 2J5, Québec, Canada.
}

\section{ABSTRACT}

Fatigue testing under fully reversed axial loading $(\mathrm{R}=-1)$ and zero-to-tension axial loading $(\mathrm{R}=0)$ was carried out on AISI 4140 gas nitrided smooth specimens. Three different treatment durations were investigated in order to assess the effect of nitriding depth on fatigue strength in high cycle fatigue. Complete specimens characterization, i.e. hardness and residual stresses profiles (including measurement of stabilized residual stresses) as well as metallographic and fractographic observations, was achieved to analyse the fatigue behaviour.

Fatigue of the nitrided steel is a competition between a surface crack growing in a compressive residual stress field and an internal crack or "fish-eye" crack growing in vacuum. Fatigue life increases with nitriding depth until surface cracking is slow enough for failure to occur from an internal crack. Unlike bending, in axial fatigue "fish-eye" cracks can initiate anywhere in the core volume under uniform stress. In these conditions, axial fatigue performance is lower than that obtained under bending and nitriding depth may have no more influence.

In order to interpret the results, special attention was given to the effects of compressive residual stresses on surface short crack growth (closure effect) as well as the effects of internal defect size on internal fatigue lives. A superimposed tensile mean stress reduces the internal fatigue strength of the nitrided steel more than the surface fatigue strength of the base metal. Both cracking mechanisms are not equally sensitive to mean stress.

\section{KEYWORDS}

gas nitriding, 4140 steel; fatigue life improvement; nitriding depth; compressive residual stresses; "fish-eye" cracks. 


\section{INTRODUCTION}

Gas nitriding is a thermochemical treatment commonly used to enhance wear, fatigue, and corrosion properties of mechanical components such as gears, crankshafts, extrusion and forging dies, valves and springs to name few. A major benefit of this surface treatment, as compared to others as for instance carburising, is the ability to harden parts without or with minimal distortion. Gas nitriding is generally performed between $500^{\circ} \mathrm{C}$ and $580^{\circ} \mathrm{C}$ in an ammonia-containing atmosphere. It involves the adsorption of atomic nitrogen into the material surface and its subsequent diffusion. Consequently, a nitrogen concentration gradient is formed. When the concentration exceeds the maximum solubility of the $\alpha-\mathrm{Fe}$ matrix, nitrogen combines with $\mathrm{Fe}$ and alloying elements, such as $\mathrm{Cr}, \mathrm{Al}$ or $\mathrm{Mo}$, to form nitride precipitates ${ }^{1}$. Nitriding, as well as carburising, shot peening or induction hardening, increases surface hardness and generates compressive residual stresses. Here, the unaffected core restrains the volume expansion caused by both the introduction of nitrogen in $\alpha$-Fe matrix and the precipitation of nitrides. The compressive residual stresses in the case are balanced by tensile residual stresses in the core.

Surface hardening and compressive residual stresses usually increase the fatigue limit of steels. Nitriding is beneficial in high cycle fatigue and can result in a transition from surface failure to internal failure. On the other hand, it might be disadvantageous in low cycle fatigue where cracks can initiate rapidly at the surface which has lower ductility than the core ${ }^{2}$. Most of the publications concern rotary bending fatigue testing ( $\mathrm{R}=-1$ only) and put emphasis on the increase of the fatigue limit of nitrided steels with increasing diffusion case depth ${ }^{3}$. Many authors focus on the effect of nitriding on the fatigue limit ${ }^{4}$ but they rarely investigate the whole S-N curve.

In surface hardened steels, cracks can initiate and propagate at the surface. However, if the enhanced hardness hinders initiation and the residual compressive stresses slow down propagation, the initiation site of the crack leading to failure can be shifted from the surface into the core of the specimen at internal defects; non-metallic inclusions being the most frequent ones reportedly. The S-N curve of a high strength steel can be described as a "stepwise curve" (Fig. 1) that results from the superposition of two curves"; one being associated with surface failure, and the other with internal failure. There is sometimes a marked transition between the two curves, which corresponds to the surface fatigue limit. Though the internal failure mode is normally observed at very high fatigue lives ${ }^{6-7}\left(10^{7}-10^{9}\right.$ cycles), surface strengthening can shift the transition between surface and internal failure modes (point A) at lives as short as $10^{4}-10^{5}$ cycles $^{8}$ (point B). Unlike surface cracks, internal cracks are not affected by surface defects and environment factors (e.g. corrosion, hydrogen embrittlement, or simply air oxidation). Actually, internal cracks initiation and growth depend on the core properties only, e.g. hardness and flaw size ${ }^{9}$. 


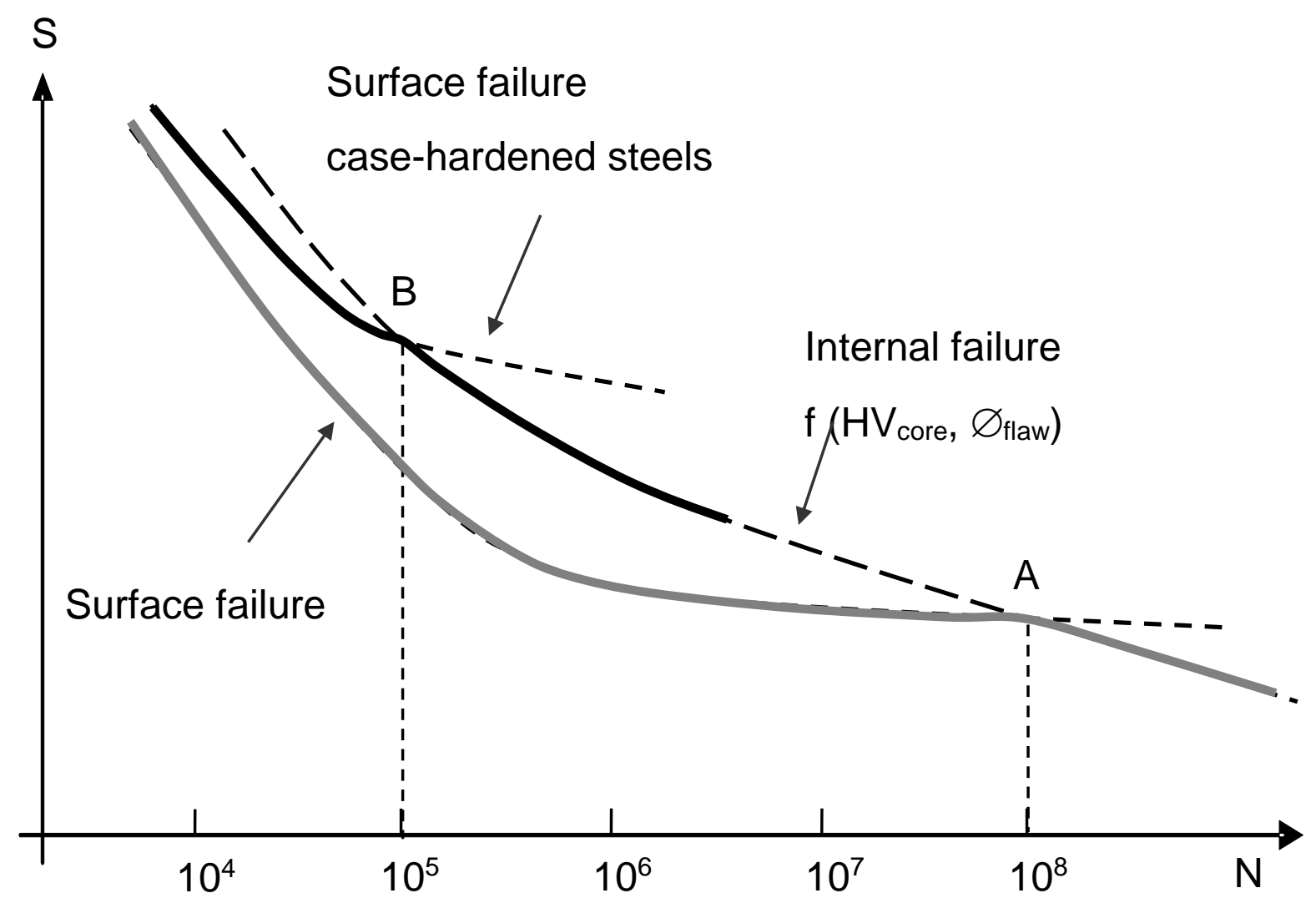

Figure 1: "Stepwise" S-N curves (thick lines)

In bending of smooth specimens, internal cracks initiate below the nitrided case near the case-core interface and the fatigue strength continuously increases with increasing nitriding depth $^{3}$. This is due to the combined effects of the stress gradient and the material volume subjected to high stress. When the nitriding depth increases, a higher bending stress has to be applied at the surface in order to produce a subsurface stress level high enough to initiate a fatigue crack. Besides, the highly stressed volume is much smaller in bending than in axial tension ${ }^{10}$. In bending, it is confined below the diffusion layer and it has been observed that the deeper the nitrided layer, the closer to the diffusion case the initiation site ${ }^{3}$. Since the probability to have a large defect decreases with decreasing stressed volume ${ }^{11}$, the mean fatigue strength increases.

For a given nitriding depth, the endurance limit of parts in service, which may be of larger size than laboratory specimens and hence undergo smaller stress gradients, is overestimated under bending loading of small laboratory specimens. In order to eliminate the size effect and to make a conservative estimation of fatigue performance, testing should be performed under axial loading where the whole specimen is subjected to a uniform stress and where the largest internal defects of the material are likely to be present. The objective of the present study is to determine the effects of gas nitriding on the axial fatigue behaviour and properties of an AISI 4140 steel.

Based on the above discussion, the main questions that need to be answered are the following:

(a) How significant is the improvement in fatigue life under axial loading?

(b) How do material (nitriding depth, inclusions) and loading (stress range and stress ratio)

parameters influence the mechanisms of surface and internal cracking? 
(c) How to explain and predict the benefits of nitriding on fatigue life?

\section{EXPERIMENTAL CONDITIONS}

\section{Specimens preparation}

The base metal is a standard medium carbon CrMo low-alloy steel (AISI 4140) supplied in the hot-rolled condition. Bars of $1 \frac{1 / 4}{4}$ inch in diameter were austenized at $815^{\circ} \mathrm{C}$, then quenched in oil and tempered in salt bath at $580^{\circ} \mathrm{C}$ for $1 \frac{1 / 2}{2}$ hour to a hardness of $36 \mathrm{HRC}$.

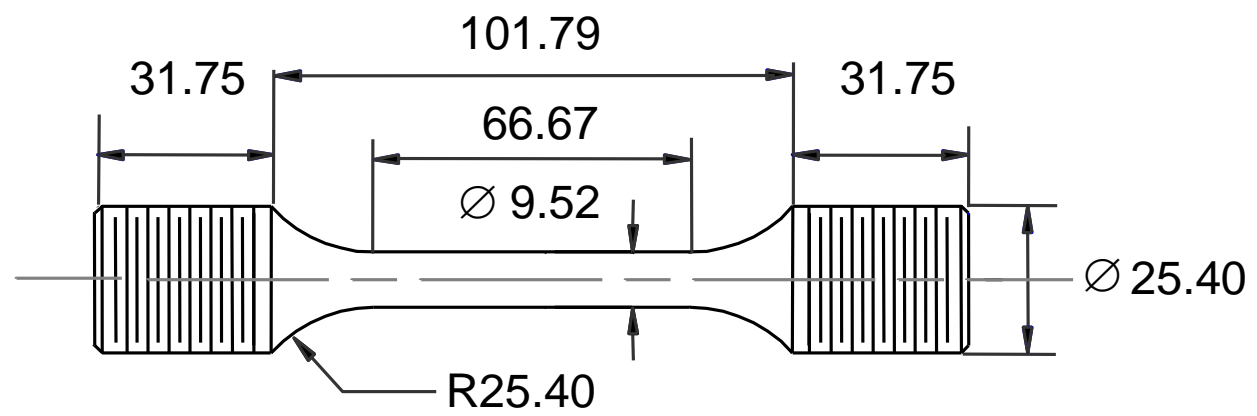

a)

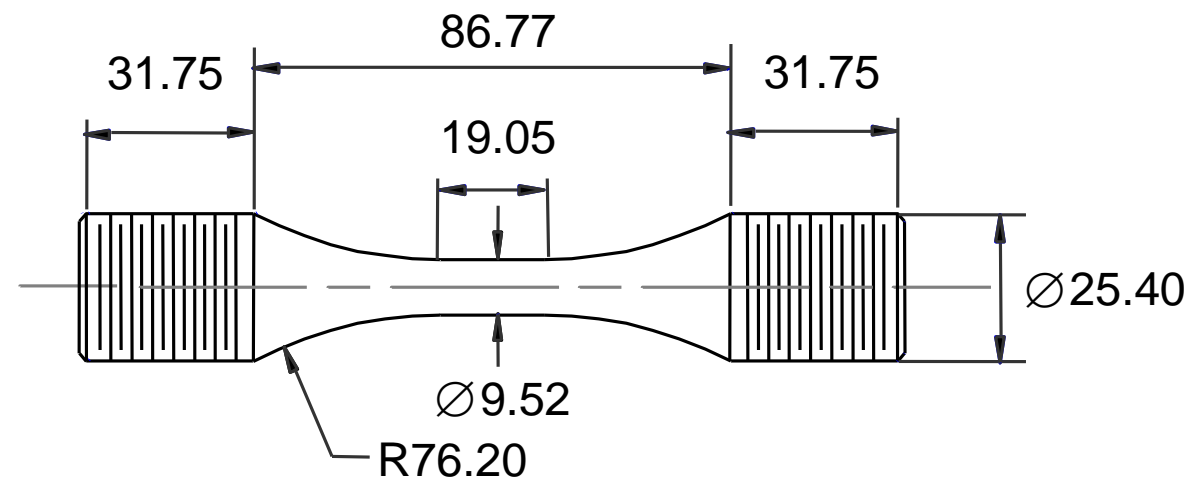

b)

Figure 2: Geometries of (a) tensile and (b) fatigue specimens (dimensions in mm)

Tensile and fatigue specimens were machined according to ASTM standards E8 and E466. Their dimensions are given in Figure 2. Fatigue specimens with uniform cross section were preferred to hourglass specimens because of their large and uniformly stressed volume, which increases the probability to have larger internal defects and decreases the risk to overestimate fatigue strength. A careful longitudinal polishing was performed to remove circumferential scratches along the gauge length. The specimens threaded ends were protected with stop-off paint before nitriding to prevent them from being treated and to avoid early failure of brittle threads.

Gas nitriding was performed by a controlled process ${ }^{12}$ where the nitriding potential was continuously adjusted to control the white layer thickness and to obtain reproducible results. The nitriding potential value was selected to give a reasonable hardness increase in the diffusion case and a white layer deep enough to provide wear resistance. In order to study the effect of the diffusion layer thickness upon material properties, three different treatment durations (i.e. 2.5, 14 and 58 hours) were performed, with temperature and nitriding potential kept constant. The three series of specimens will be referred hereafter as to shallow, medium and deep case respectively. 


\section{Residual stress measurement}

Extra specimens identical to the fatigue specimens were devoted to X-ray residual stress measurement. Macro-residual stress determination was realized according to the " $\sin ^{2} \Psi$ " method $^{13}$ using the $\mathrm{CrK}_{\alpha}$ radiation $(\lambda=2.291 \AA)$ and a $1 \mathrm{~mm}$-round collimator. With this method, a strain is measured from the deformation of the crystal lattice. The residual stress related to this strain is calculated assuming linear elastic distortion. The Martensite $\{211\}$ diffracted line of the $\alpha$-Fe matrix $\left(2 \theta=156.1^{\circ}\right)$ was recorded at many sample orientations (from 18 to $22 \psi$ angles) with acquisition time ranging from $15 \mathrm{~s}$ to 40 s per angle. To obtain a residual stress profile in depth, electrolytic polishing was performed to decrease the specimen radius progressively. Residual stresses were corrected for redistribution due to material removal as per SAE J784a standard. They were obtained in the longitudinal direction of the specimen, which was considered the most important one with respect to the fatigue properties. Other measurements were also carried out on pre-cycled specimens to give an estimate of residual stress relaxation.

\section{Tension testing \& Fatigue testing}

Tension testing was performed under stroke control according to ASTM standard E8. High cycle axial fatigue testing was performed under load control according to ASTM standard E466. Special care was given to ensure a good alignment of the grips in order to minimize bending. Specimens were subjected to constant amplitude fully reversed loading $(\mathrm{R}=-1)$ at a frequency of $10 \mathrm{~Hz}$ under the three nitriding conditions. Testing was also performed under zero-to-tension loading $(\mathrm{R}=0)$ on medium case specimens. Quenched and tempered base metal specimens were tested as a reference. Fracture surfaces were observed under an optical stereomicroscope and a scanning electron microscope (SEM) equipped with an X-ray energy dispersive spectrometer (EDS).

\section{EXPERIMENTAL RESULTS}

\section{Metallographic observation and hardness profiles}

Control samples were coated with an electrolytically deposited nickel layer to avoid damaging of the white layer during polishing. Nital etching was used to distinguish the white layer from the diffusion layer. The white layer consists in an iron nitride layer where some porosity can be observed at the very surface; its depth ranges from $1 \mu \mathrm{m}$ to $10 \mu \mathrm{m}$. The diffusion case beneath the white layer shows the same tempered martensitic microstructure as the base metal. The nitride precipitates are not discernible under an optical microscope.

Microhardness profiles are reported in Figure 3(a). Intersection of the hardness profiles in the nitrided steel with the matrix hardness $(355 \mathrm{HV})$ gives three total diffusion depths of approximately $400 \mu \mathrm{m}, 600 \mu \mathrm{m}$, and $1000 \mu \mathrm{m}$. Microhardness profiles show a peak up to $640 \mathrm{HV}$ near the surface for the shallow and medium case depths but a markedly smaller value for the large case depth, about 530HV. After prolonged nitriding, the maximum hardness decreases due to a discontinuous precipitation at grain boundaries and to a coarsening of the continuous $\mathrm{CrN}$ precipitates ${ }^{14}$. 
a)

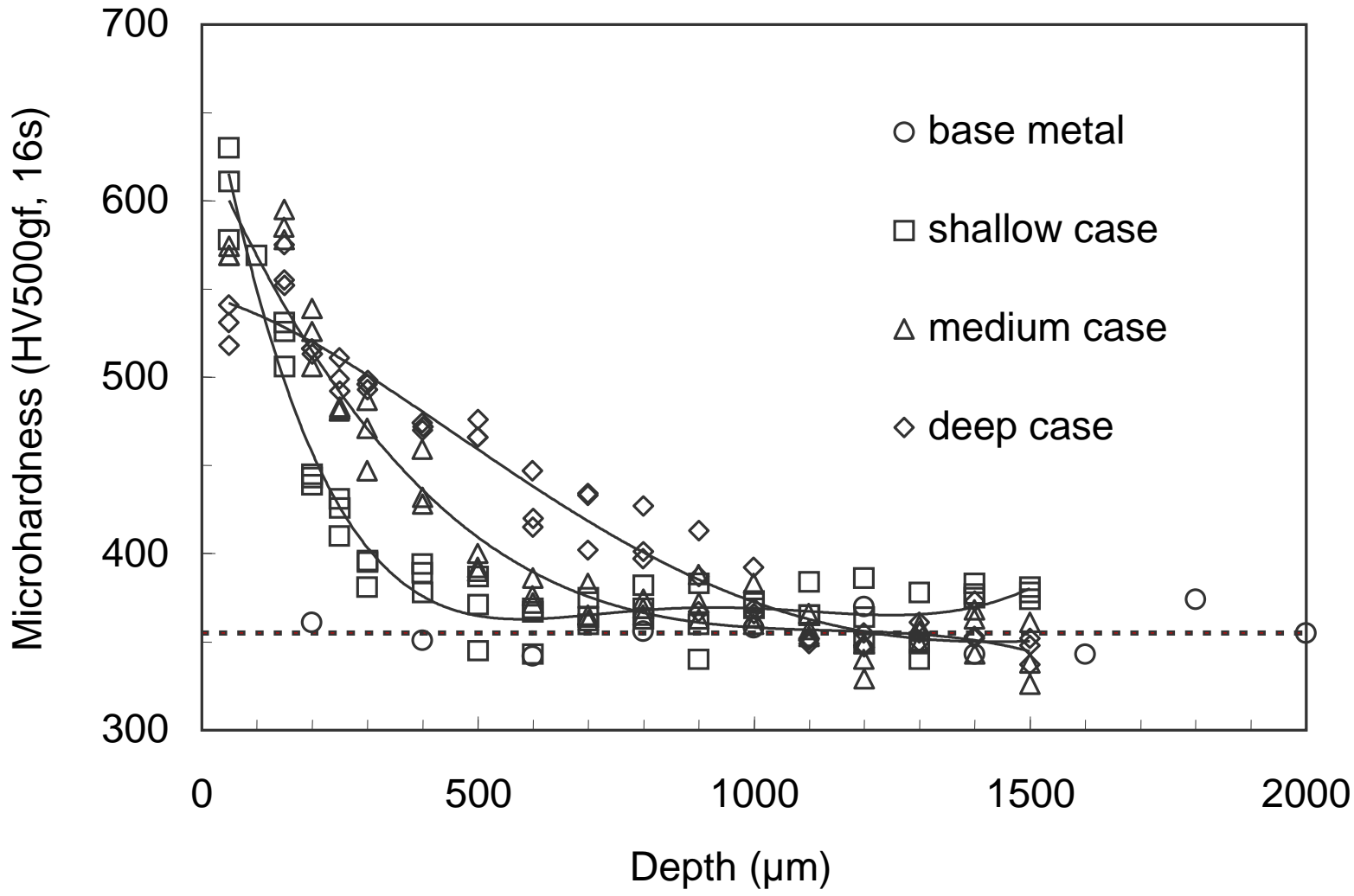

b)

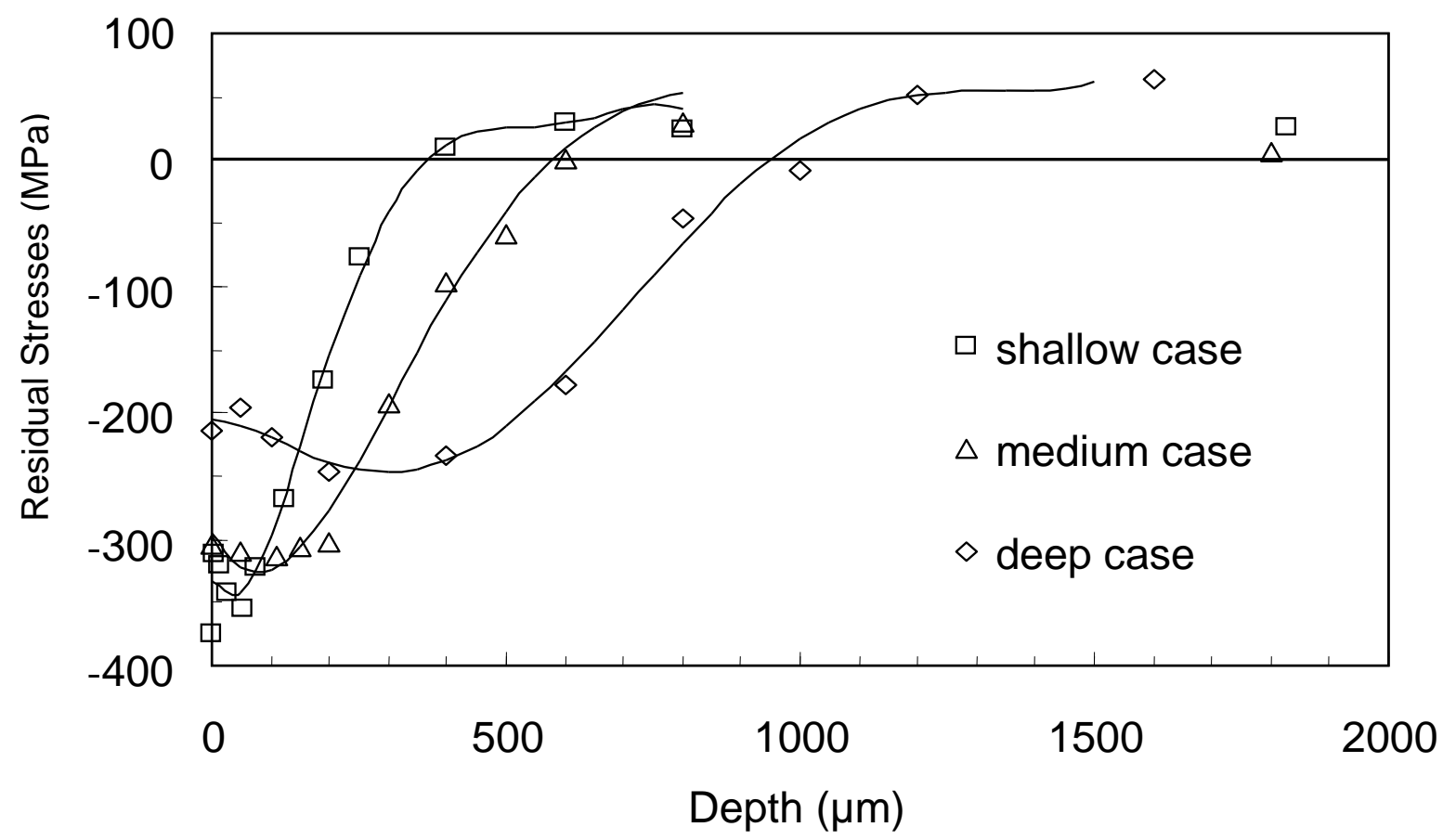

Figure 3: (a) Microhardness and (b) residual stress profiles for the three case depths

\section{X-Ray measurement of residual stresses}

The longitudinal residual stress profiles shown in Figure 3(b) confirm that there is a compressive residual stress field at the surface balanced with a slight tensile residual stress field in the core. The transition depth from compressive to tensile residual stresses is approximately equal to the diffusion depth measured on the hardness profiles. However, the 
compressive peak decreases and moves toward the core with increasing nitriding depth.

During long nitriding treatments, the surface residual stresses are partially relaxed due to the discontinuous precipitation and to the subsequent segregation or diffusion of the excess nitrogen, which are also responsible for the lower hardness ${ }^{14}$. Furthermore, nitriding of deep layers consumes nitrogen, which is no more available to form precipitates at the surface.

Residual stress profiles measured on a medium case sample after 1000 fatigue cycles at $\pm 600 \mathrm{MPa}$ and at $\pm 700 \mathrm{MPa}$ show no evidence of residual stress relaxation.

\section{Tensile testing}

The monotonic tensile strength of the nitrided steels is not very different from that of the base metal but the ductility is drastically reduced as shown in Figure 4. The tensile yield strength of the nitrided steel slightly increases with increasing case depth. Series of secondary circular cracks developed in the case during tensile deformation as previously noticed by Mencik $^{15}$. This caused the extensometer to record discontinuous curves after the yield point.

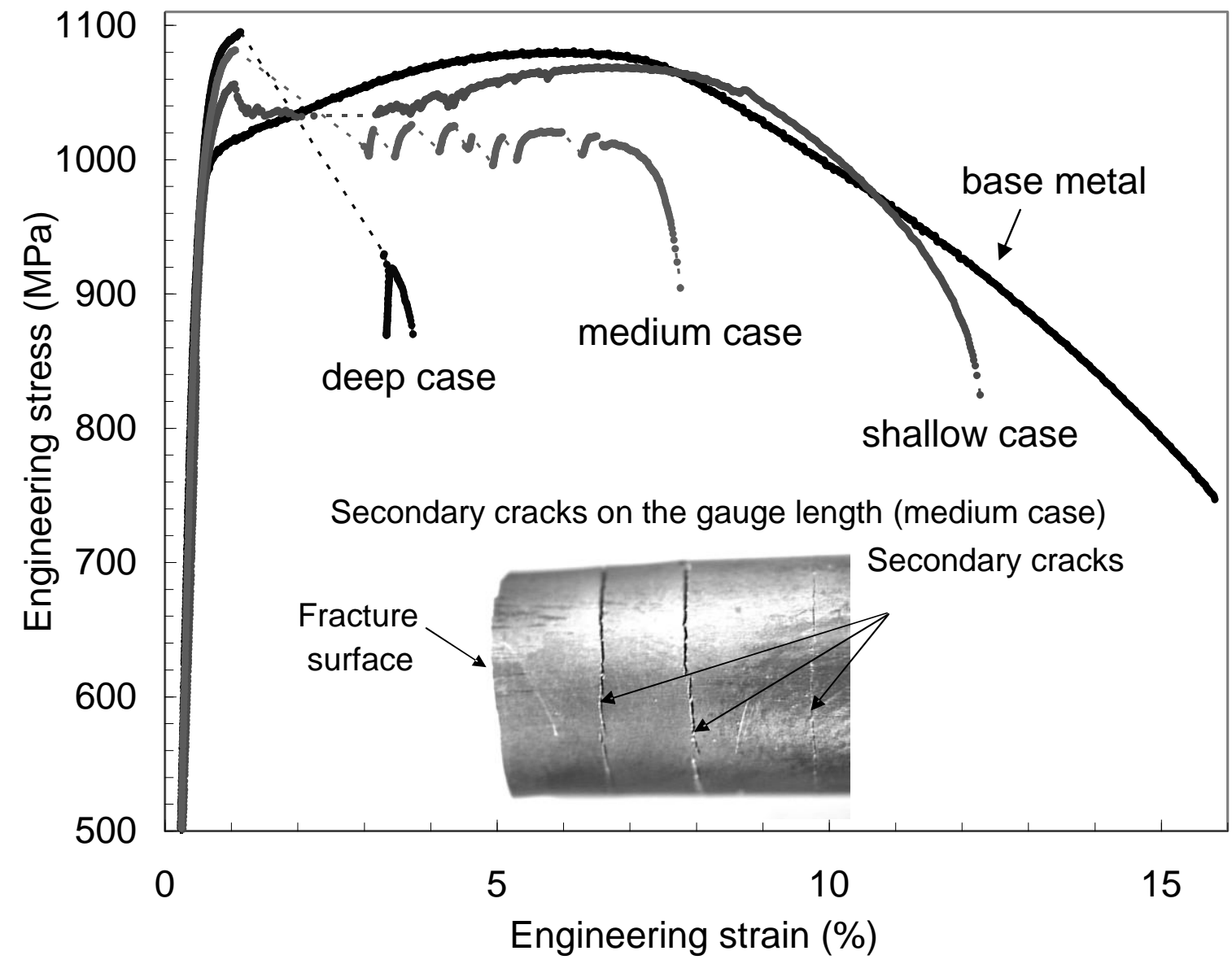

Figure 4: Tensile testing curves

\section{Push-pull fatigue testing}

$\mathrm{S}-\mathrm{N}$ data are plotted in Figure 5. All tests were conducted at a $10 \mathrm{~Hz}$-frequency except at $700 \mathrm{MPa}$ where it was necessary to reduce the frequency at $1 \mathrm{~Hz}$ to avoid specimen heating and premature fracture. 


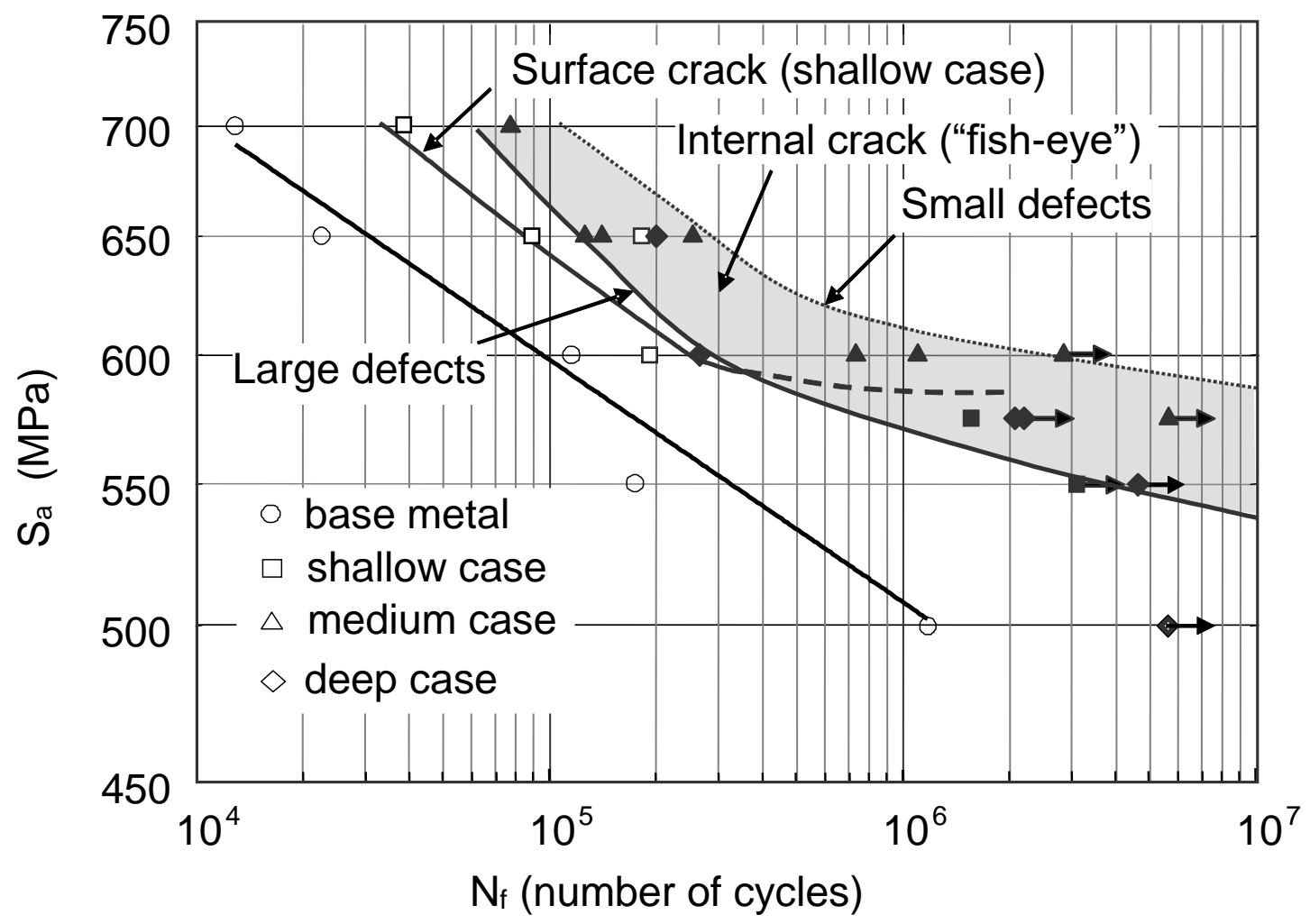

Figure 5: S-N data under fully reversed loading (open symbol: surface failure; full symbol: "fisheye" crack)

Nitriding improves high cycle fatigue life whatever the case depth. This improvement increases with decreasing stress level. With respect to the quenched and tempered base metal, the fatigue life improvement ratio ranges from 3 to 6 at $700 \mathrm{MPa}$ and, from 10 to more than 40 at $575 \mathrm{MPa}$. Shallow case always gives the lowest improvement while deep case never gives better results than medium case and can even give shorter lives at low stress levels, i.e. at $575 \mathrm{MPa}$ and $600 \mathrm{MPa}$.

The final fracture of nitrided steels results from the initiation and the propagation of a surface crack (Fig. 6(a)) or from the propagation of a circular "fish-eye" crack at an internal defect (Fig. 6(b)), e.g. an inclusion or a microstructural discontinuity. Medium and deep case specimens always failed from the interior. Shallow case specimens failed from the surface except at the lowest stress levels below 600MPa. All the internal failures have been plotted inside a scatter band in Figure 5. The lower bound is associated to inclusions from $20 \mu \mathrm{m}$ to $45 \mu \mathrm{m}$ in size and the upper bound to smaller microstructural discontinuities. At a given life of 200,000 cycles, the fatigue strength improvement can be as small as $10-15 \%$, which is much lower than values of $30-50 \%$ reported in rotary bending ${ }^{3}$.

Though low cycle fatigue was not evaluated in this study, it is expected that nitriding may be no more beneficial below $10^{4}$ cycles. In this regime, the fatigue life depends on the material ductility. As a result, the brittle case will fail soon and a surface crack will propagate rapidly through the core. This can cause a reduction in fatigue life compared with the base metal as shown by Jones and al. ${ }^{16}$. The tensile test can be regarded as an extreme low cycle condition with the broken case acting as a notch on the ductile core. 
a)

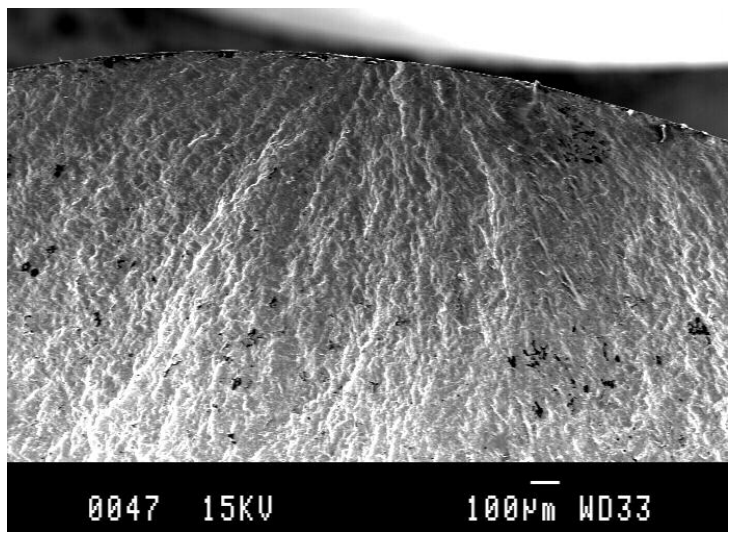

b)

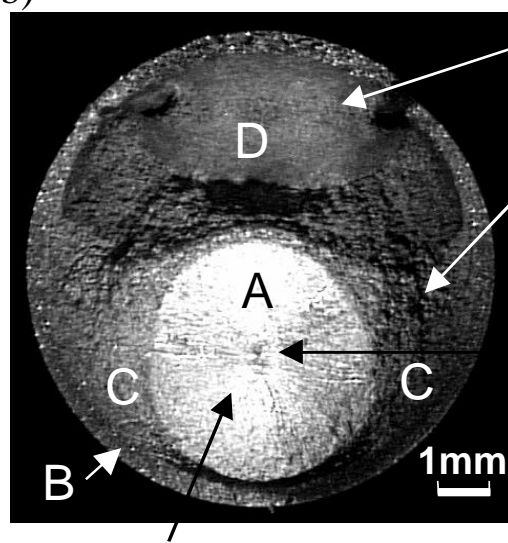

Slow propagation
Final ductile fracture Rapid propagation

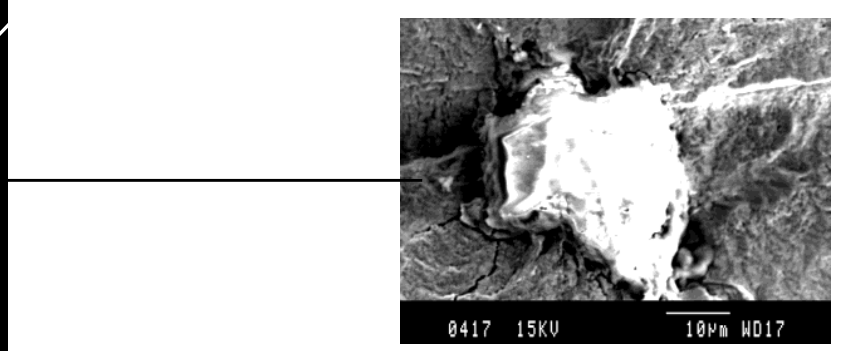

Inclusion at the centre of the fish-eye $(\mathrm{Al}, \mathrm{O}, \mathrm{Mg}, \mathrm{Ca}, \mathrm{S})$

\section{Figure 6: (a) Surface crack (shallow case) (b) "Fish-eye” crack (medium case)}

The "fish-eye" cracks observed in the present study can initiate anywhere in the core, which is under uniform loading with only slight tensile residual stresses. Figure 7 includes data from the present study as well as data ("deep case 2") obtained from preliminary testing of deep nitrided specimens made with a slightly softer 4140 base metal (320HV instead of $355 \mathrm{HV})$. Figure 7 shows that the internal initiation sites are evenly distributed from below the diffusion layer to the centre of the specimen for both medium and deep cases. No correlation was found between the "fish-eye" initiation depth and the life improvement.

X-ray analysis of the inclusions at the centre of the "fish-eyes" reveals that they consist of complex oxides such as compounds of $\mathrm{Al}, \mathrm{Mn}$, and $\mathrm{Ca}$. Initiation at an inclusion first takes place by decohesion of the inclusion from the matrix, then a crack grows radially from the inclusion. A "fish-eye" crack failure consists in four fractographic regions visible in the naked eye as shown in Figure 6. First, the "fish-eye" itself is a white circular spot (region A), which is, according to Murakami et al. ${ }^{17}$, characteristic of a slow crack growth under a low level of oxygen. The nitrided case (region B) always presents a flat failure surface with, occasionally, some tiny signs of fatigue crack growth from the case towards the core (region C) depending on the initiation depth. A dark and rough area (region D) results from the final ductile fracture.

Macroscopic features are different whether the initiation site is located close to the diffusion layer or not, as already discussed by Limodin et al. ${ }^{18}$. Nevertheless, though the "fish-eye" size ranges from a few hundred micrometers to a few millimetres, the initiation 
depth does not influence the fatigue life (Fig. 7). Thus, most of the internal fatigue life is spent near the "fish-eye" centre.

\section{- shallow case $\Delta$ medium case $\bullet$ deep case $\diamond$ deep case 2}

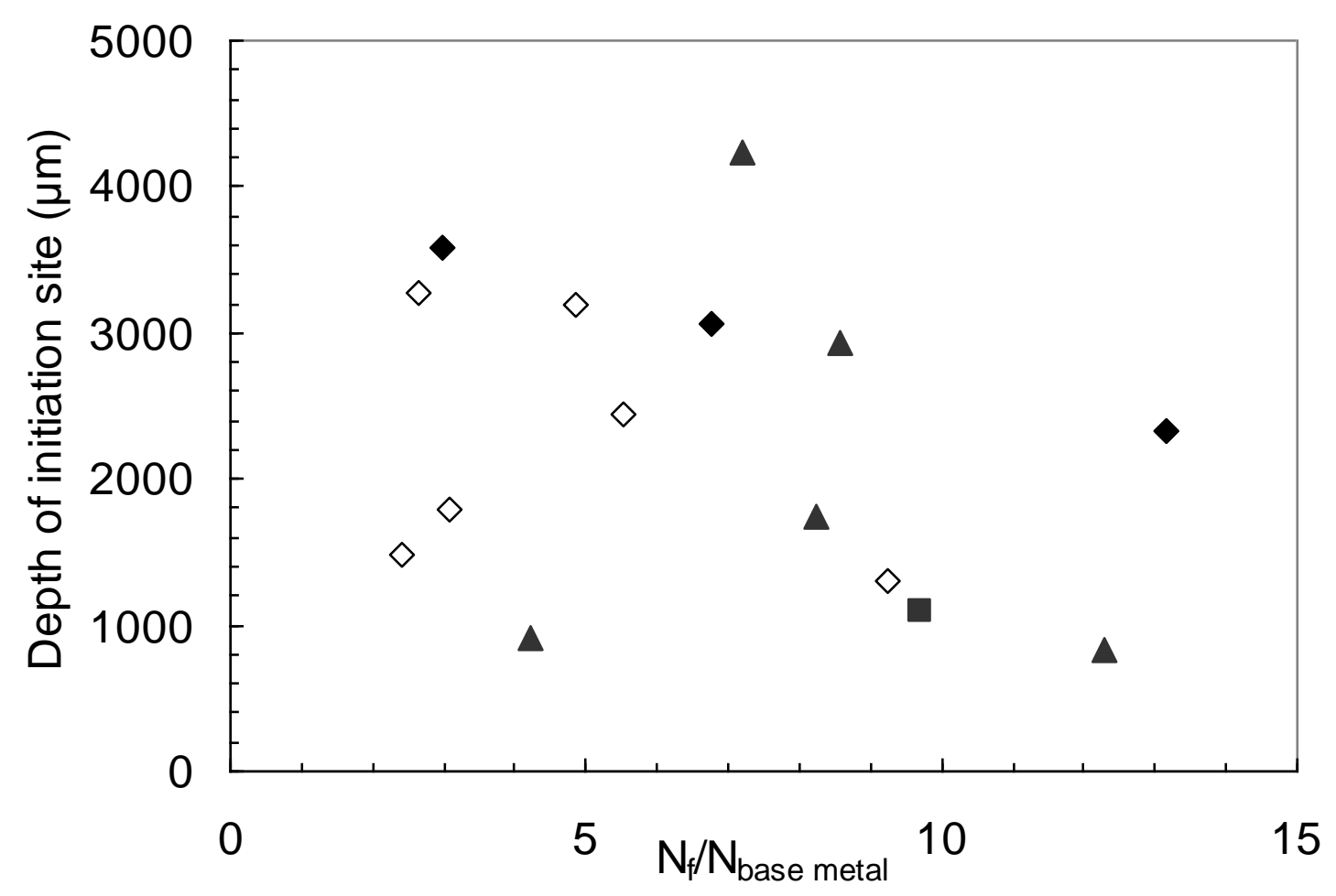

Figure 7: "Fish-eyes" initiation depth versus fatigue life improvement ratio

\section{Zero-to-tension fatigue testing}

To assess the effect of a tensile mean stress on the fatigue strength of nitrided steel, medium case specimens and base metal specimens were submitted to zero-to-tension loading. Results for stress amplitudes ranging from $425 \mathrm{MPa}$ to $525 \mathrm{MPa}$ are reported in Figure 8. Nitriding still improves fatigue life as compared to the reference steel as long as the maximum applied stress is less or equal to $1000 \mathrm{MPa}$. This improvement is associated to an internal failure mode and it ranges from 3 to 4 times the reference fatigue life. Comparing the stress amplitude at a given life between tension-compression loading and zero-to-tension loading, the improvement of fatigue strength due to nitriding decreases approximately by a factor of 2 . At long lives ( $10^{6}$ cycles), it ranges between $17 \%$ and $23 \%$ without mean stress while it becomes lower than $10 \%$ under zero-to-tension loading. 


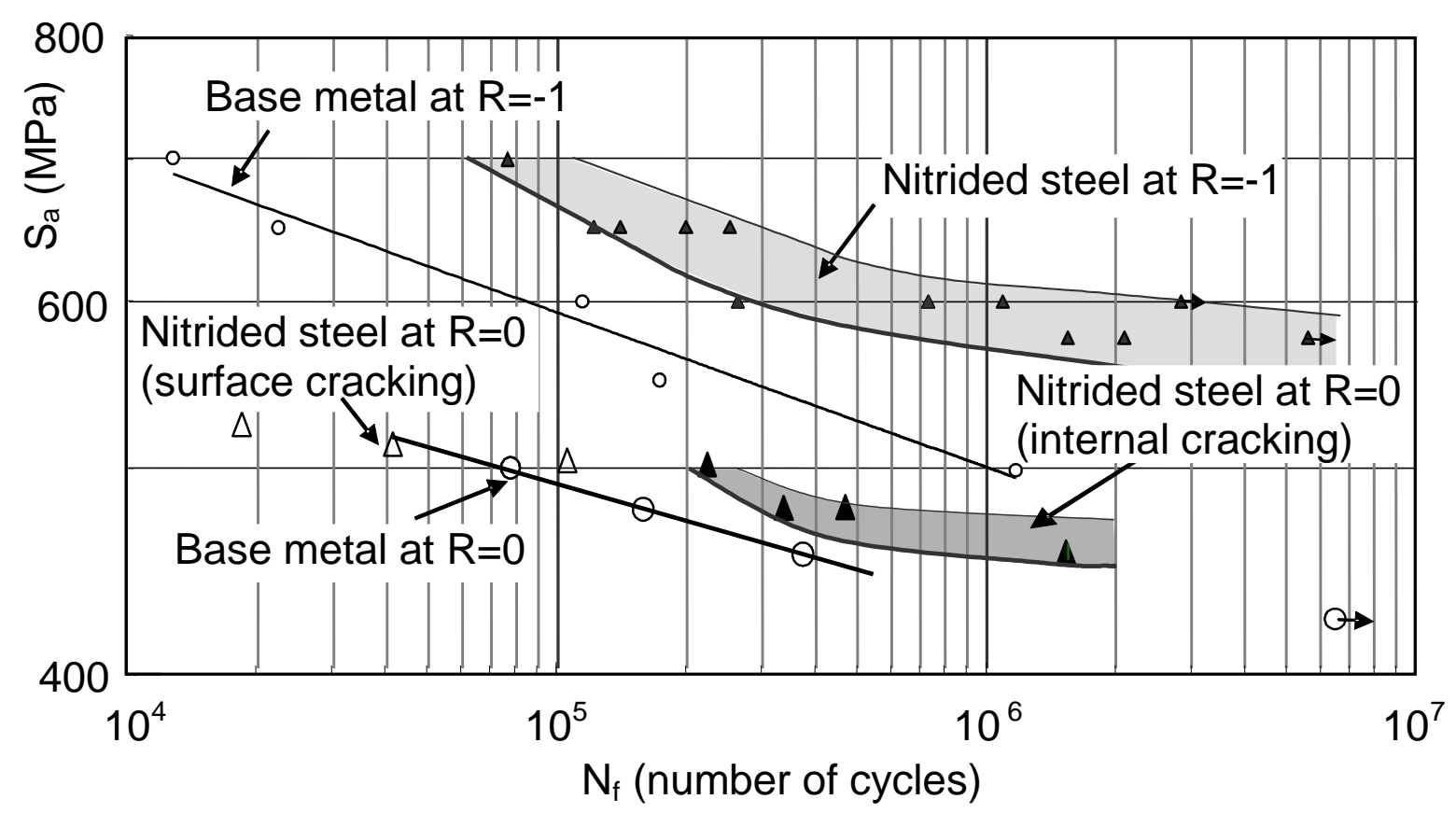

Figure 8: $S$-N curves for zero-to-tension loading (medium case specimens) as compared to fully reversed loading

For maximum applied stress larger than 1000MPa, nitrided specimens failed from a surface mechanism and it was not possible to measure any enhancement in fatigue life. At these high stress levels close to the yield stress, the reference specimens failed by cyclic creep.

\section{DISCUSSION}

\section{Monotonic tensile properties}

The yield strength of nitrided specimens is still controlled by the bulk core properties because surface hardening only affects a thin surface layer as compared to the total section of the specimen. However, once the thin nitrided layer is broken, it acts as a notch or a stress raiser on the remaining un-broken part, i.e. the soft-core material, which fails without much ductility $^{2}$. Some authors even defined the hardened layer as a metallurgical notch ${ }^{19}$. Jack ${ }^{20}$ pointed out that the situation is that of a single ductile fibre, i.e. the core, in a brittle matrix, i.e. the nitrided case. Because of the residual tensile stresses in the core, the actual core stress exceeds its yield strength value early in the test but the core is constrained from yielding by the elastic case. As a result, the composite deforms elastically until the strain is sufficient to break the case. The difference between Poisson's ratios in the case $(v=0.3)$ and in the core $(v=0.5)$ introduces stress triaxiality at the interface ${ }^{21}$. The core tends to contract more than the case and transverse stresses are generated in the radial and circumferential directions.

Secondary cracks found on the specimen surface (Fig.4) are often observed when testing metals with surface coating or treatment. Mencik ${ }^{15}$ explained that, when a surface treated specimen is subjected to a tensile loading, shear stresses are induced at the interface between the coating and the substrate (i.e. the case and the core). At a free edge (i.e. the first crack in the case), no stress acts in the case and the entire load is carried out by the core. Some distance away from this crack, the load is carried by the case as well as the core. Near the 
edge, shear stresses appear at the interface and cause the tensile stresses to increase gradually in the case until the case strength is reached. Then failure of the case occurs and it creates a new edge and so on. This phenomenon generates an array of circumferential cracks along the gauge length as shown in Figure 4.

\section{"Fish-eye" fatigue cracking: effect of internal defect size}

High cycle fatigue of nitrided steel is a competition between two fracture mechanisms slower than surface cracking in the base metal. Fracture results either from an internal crack propagating in vacuum or from a surface crack whose growth is delayed by nitriding. The fatigue life is that corresponding to the fastest mechanism.

Under fully reversed loading, medium and deep case nitriding were sufficient for surface cracking to be slower than initiation and growth of an internal crack whatever the stress level. There is no evidence that internal fatigue life depends on nitriding depth. Globally, neither the deep case nor the medium case has given better results (Figs. 5-7). On the other hand, the internal fatigue life depends on the matrix properties and on the size of the worst defect that exists in the stressed volume. Internal cracking induces a large scatter with the shortest lives corresponding generally to the largest defects as shown on Table 1. The longest lives were often obtained for "fish-eyes" initiated from a microstructural discontinuity smaller than an inclusion (no inclusions or defects can be observed under SEM). The life scatter between specimens, which failed from "fish-eye" cracks at a same stress level, can be partially attributed to the size of the defect leading to fracture.

Table 1: Internal defect sizes at the origin of "fish-eye" cracks

\begin{tabular}{|l|l|l|l|}
\hline $\begin{array}{l}\mathrm{S} \\
{[\mathrm{MPa}]}\end{array}$ & $\begin{array}{l}\text { Nitriding } \\
\text { depth }[\mu \mathrm{m}]\end{array}$ & $\begin{array}{l}\text { Inclusion } \\
\text { “Varea" }[\mu \mathrm{m}]\end{array}$ & $\mathrm{N}_{\mathrm{f}}$ cycles] \\
\hline 575 & 400 & 38 & $1.6 \times 10^{6}$ \\
575 & 1000 & 0 & $2.1 \times 10^{6}$ \\
\hline 600 & 1000 & 27 & $2.7 \times 10^{5}$ \\
600 & 600 & 0 & $7.3 \times 10^{5}$ \\
600 & 600 & 0 & $1.1 \times 10^{6}$ \\
\hline 650 & 600 & 45 & $1.3 \times 10^{5}$ \\
650 & 1000 & 0 & $2.0 \times 10^{5}$ \\
650 & 600 & 27 & $2.5 \times 10^{5}$ \\
\hline
\end{tabular}

Varea=0: no inclusion or defect is observed under SEM

In Figure 5, internal fatigue lives stand between two borderlines: a S-N curve for small defects, i.e. microstructural discontinuities, and a S-N curve for large defects, i.e. large nonmetallic inclusions. We have also drawn a S-N curve corresponding to surface fatigue lives of shallow case specimens. Shallow case nitriding was efficient at $575 \mathrm{MPa}$ only. At higher 
stress levels, the shallow case delays crack initiation and growth at the surface, but the life improvement is small. The surface fatigue life is lower than the internal fatigue life.

\section{Surface fatigue cracking: effect of residual stress field}

Medium and deep case specimens did not fail from the surface even at the highest stress levels (Fig. 5). This implies that their surface fatigue lives are, at least, larger than the measured internal fatigue lives. The ratio of surface fatigue lives between medium case and shallow case is more than one order of magnitude at 600MPa (Fig. 5). This improvement cannot correspond to any delay in surface crack initiation. Actually, shallow case and medium case specimens should have the same initiation life because they both have the same hardness and residual stress profiles until $100 \mu \mathrm{m}$ in depth (Fig. 3). They should even have a longer initiation life than deep case specimens, which have lower hardness and residual stresses at the surface. The difference in fatigue lives can be attributed to short crack propagation through the nitrided case. Short crack propagation can be slower in a compressive residual stress field that extends deeper into the material.

In the short crack regime, even above the microstructural scale, LEFM is theoretically not valid due to the high nominal stress levels necessary for short crack propagation. However, a previous study ${ }^{22}$ where surface cracks were monitored between $0.1 \mathrm{~mm}$ and $0.5 \mathrm{~mm}$ in depth has revealed that short crack growth rates are reasonably well consolidated with LEFM long crack growth rates when using the effective SIF range $\left(\Delta \mathrm{K}_{\mathrm{eff}}\right)$ to account for crack closure effects. Measurements of crack opening level under fully reversed loading $(R=-1)$ showed that the effective fraction of the SIF range, $\mathrm{U}=\Delta \mathrm{K}_{\mathrm{eff}} / \Delta \mathrm{K}$, is as small as 0.3 for LEFM cracks $\left(\mathrm{K}_{\mathrm{op}}=+0.4 \mathrm{~K}_{\max }\right)$ while it can reach about 0.55 for short cracks in a stress relieved metal $\left(\mathrm{K}_{\mathrm{op}}=-0.1 \mathrm{~K}_{\mathrm{max}}\right)$.

Figure 9 schematically represents the decreasing effect of the residual SIF $\left(\mathrm{K}_{\text {res }}\right)$ in the nitrided steel upon the effective SIF range ( $\left.\Delta \mathrm{K}_{\text {eff }}\right)$. The compressive residual stresses are superimposed to the applied stresses. As a result, the SIF range available to propagate a crack decreases and the propagation life increases. The evolution of the residual stress intensity factor $\left(\mathrm{K}_{\text {res }}\right)$ with crack depth has been computed by applying the initial residual stress distribution along the crack faces as suggested by Kim et al. ${ }^{23}$. The resulting effect upon the crack driving force is presented in Figure 10, where the effective fraction (U) is plotted versus crack depth, assuming that the absolute opening level remains close to zero $\left(\mathrm{K}_{\mathrm{op}}-\mathrm{K}_{\mathrm{res}}=-0.1 \mathrm{~K}_{\mathrm{max}}\right)$. The effective fraction $\mathrm{U}$ decreases to a minimum, then it gradually increases to the value of 0.55 at large crack depths. Because of stress redistribution, residual stresses at the crack tip remain compressive, even beyond the point where the initial residual stresses change sign.

Short crack propagation lives were computed for the base metal and the three nitriding conditions by integrating a da/dN- $\Delta \mathrm{K}_{\text {eff }}$ propagation law from $\mathrm{a}_{0}=0.1 \mathrm{~mm}$ to $\mathrm{a}=0.8 \mathrm{~mm}$. A hardness-dependent propagation law was used based on a paper from Kato et $\mathrm{al}^{24}$. The lives computed for the nitrided steels are much larger than those for the base metal. The fatigue life improvement ratio computed for the medium case exceeds one order of magnitude. However, the short crack model cannot predict the low performance of shallow case specimens whatever adjustments are made in the propagation law coefficients. The lives computed for the shallow case are hardly lower than those for the medium case. Further the model predicts that the deep case would have the worst life improvement. 


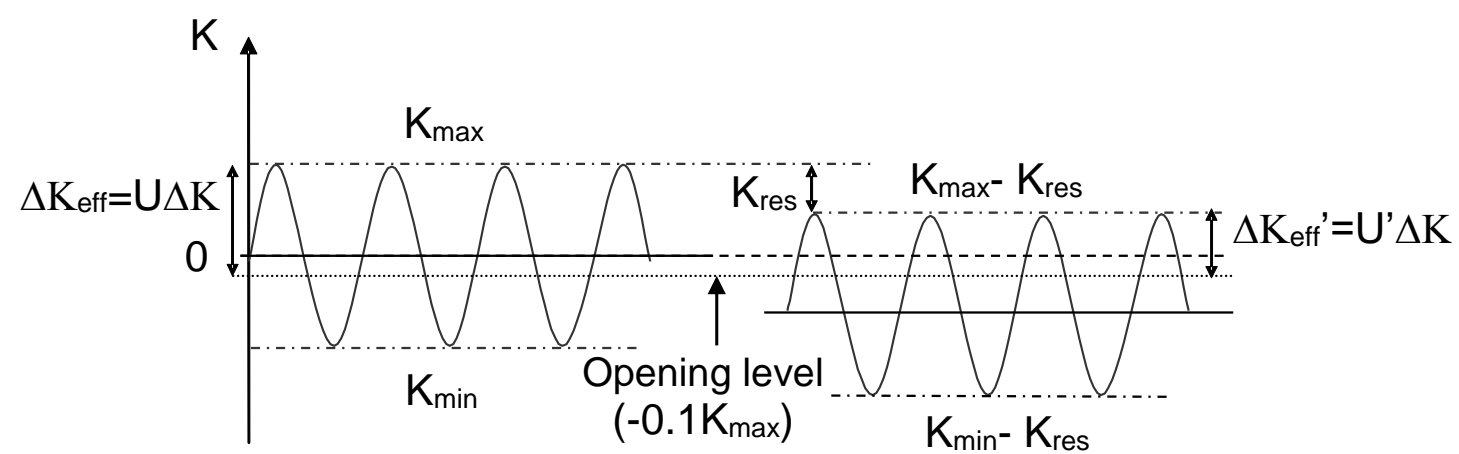
Short crack without
Short crack in
residual stresses nitrided steel

Figure 9: Effect of a compressive residual SIF upon the effective SIF range.

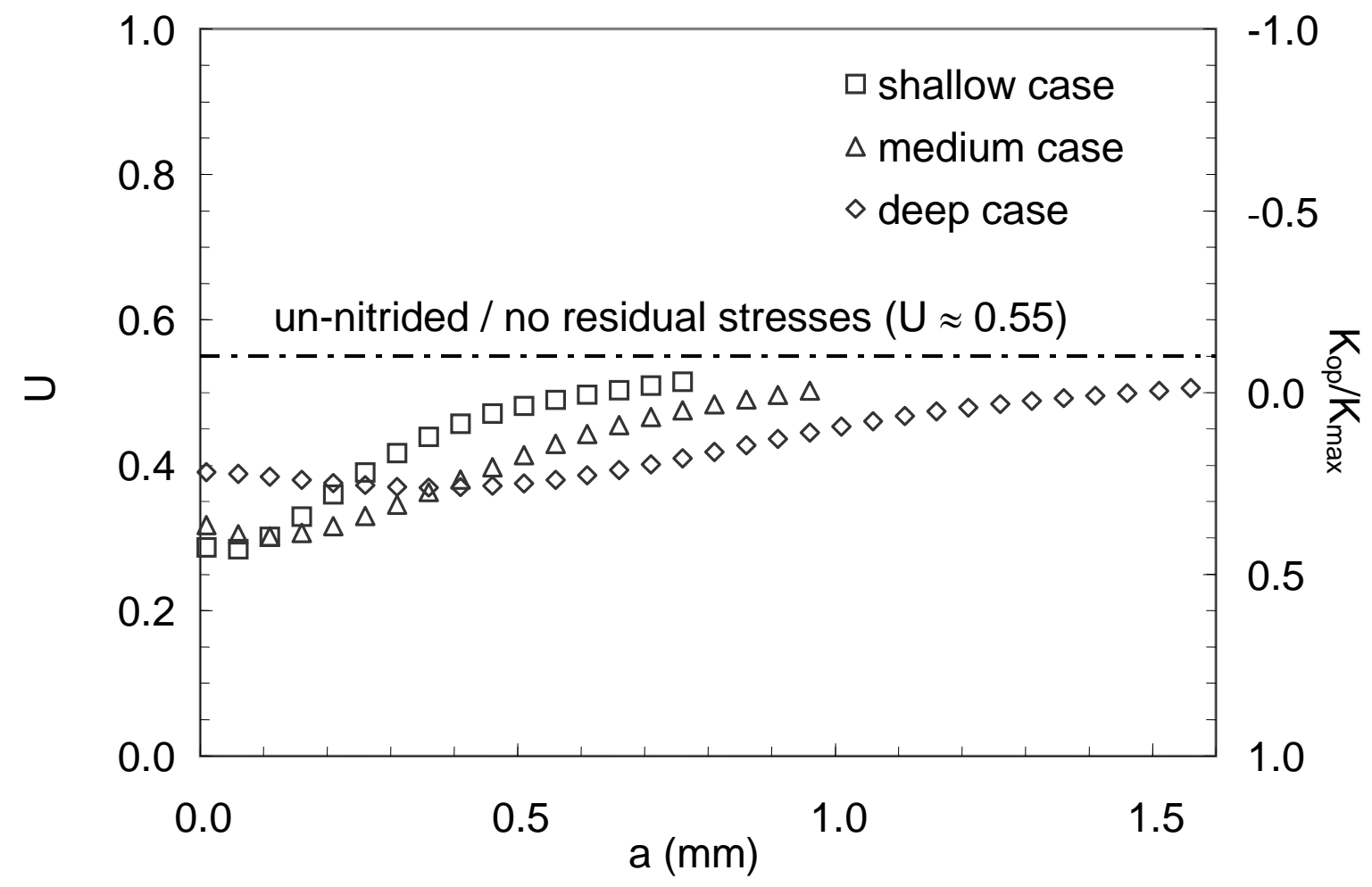

Figure 10: Effective fraction of the SIF range versus crack depth for the three case depths $(S=650 M P a)$

\section{Mean stress effect on fatigue strength}

Except at high stress levels close to the yield strength, the medium case specimen under zero-to-tension loading has its surface strengthened enough to always fail by internal fatigue. However, the fatigue strength improvement decreases approximately by a factor of two with respect to fully reversed loading (Fig. 8). Nitrided steel failing by internal fatigue appears to be more sensitive to a superimposed mean tensile stress than the base metal. The decrease in stress amplitude between fully reversed loading and zero-to-tension loading is about $15 \%$ for the base metal, while it lies between $21 \%$ and $25 \%$ for the nitrided steel. Using $\mathrm{R}=-1$ data and monotonic tensile strength, Gerber's model predictions give a conservative estimation of the base metal fatigue strength for $\mathrm{R}=0$ loading while they overestimate that of the nitrided steel. 
At high stress levels, i.e. when the maximum applied stress is close to the yield strength, the medium case specimen fails from the surface with no more fatigue life improvement. However, the increased yield strength of the nitrided specimen makes it less sensitive to cyclic creep than the base metal.

\section{CONCLUSIONS}

Axial high cycle fatigue testing was performed under fully reversed loading $(\mathrm{R}=-1)$ and zero-to-tension loading ( $\mathrm{R}=0$ ) on a gas nitrided AISI 4140 steel. Different treatment durations were selected to obtain three nitrogen diffusion depths and to analyse the case depth influence on axial fatigue performance. The following conclusions were drawn.

(a) Fatigue of the nitrided steel is a competition between a surface crack growing in a compressive residual stress field in a material of increased hardness and an internal crack growing in vacuum. The shallow nitrided case delays crack initiation and growth at the surface, but the improvement of surface fatigue life is small. Shallow case specimens failed from the surface, except at the lowest stress levels. For medium and deep case specimens, the improvement of surface fatigue life is large enough for failure to occur always from an internal crack. The medium case nitriding is recommended for engineering applications because the deep case nitriding is time-consuming and more expensive.

(b) Unlike bending, there is no evidence in axial fatigue that internal fatigue life depends on nitriding depth. Under uniform stress loading, "fish-eye" cracks can initiate anywhere in the core volume. At long lives, the improvement of fatigue strength ranges between $17 \%$ and $23 \%$, which is lower than that reported in rotary bending (30-50\%). The size of the internal defect from which a "fish-eye" crack propagates has a significant effect on internal fatigue lives. They are only three times larger than the base metal lives when the inclusion size reaches about $50 \mu \mathrm{m}$. A bad cleanliness of the base metal cannot give a large improvement in fatigue life. Furthermore, a superimposed tensile mean stress reduces the internal fatigue strength of the nitrided steel more than the surface fatigue strength of the base metal.

(c) A short crack propagation model, based on the effect of compressive residual stresses on crack closure, can account, at least partially, for large improvement in surface fatigue lives. However, the global influence of nitriding depth on surface fatigue lives cannot be predicted from initiation and propagation models based on the fatigue behaviour of homogeneous metals.

(d) Neither axial loading nor bending of smooth specimens are appropriate to evaluate or to predict the fatigue performance at severe notches, e.g. at the root of a gear tooth. In such a case, the stress gradient is sufficiently high so that failure from internal defects is not likely to occur. Fatigue life improvement brought by nitriding becomes fully effective when cracks must initiate and propagate at the surface.

\section{ACKNOWLEDGEMENTS}

We acknowledge Nitrex Metal for providing in-kind and monetary contributions to this study. The authors are also grateful to the Natural Science and Engineering Research Council (NSERC) of Canada for its additional financial support. 


\section{REFERENCE}

${ }^{1}$. Liliental, W.K., Tymowski, G.J. and Tarfa, N.T. (2000). Frequently encountered problems on nitrided components. In Proceedings of $12^{\text {th }}$ International Federation of Heat Treatment and Surface Engineering Congress, October 2000, Melbourne, Australia.

2. Qian, J. and Fatemi, A. (1995). Cyclic deformation and fatigue behaviour of ion-nitrided steel. Int. J. Fatigue 17 (1), 25-24.

3. Genel, K., Demirkol, M., and Çapa, M. (2000). Effect of ion nitriding on fatigue behaviour of AISI 4140 steel. Mater. Sci. Eng. A279, 207-216.

${ }^{4}$. Spies, H.J. and Trubitz, P. (1996). Fatigue of nitrided steels. In Fatigue 96 Vol.2, May 6-10 1996, Berlin, Germany, 1409-1414.

5 . Nishijima, S. and Kanazawa, K. (1999). Stepwise S-N curve and "fish-eye" failure in gigacycle fatigue. Fatigue \& Fract. Engng Mater. Struct. 22 (7), 601-607.

${ }^{6}$. Bathias, C. (1999). There is no infinite fatigue life in metallic materials. Fatigue \& Fract. Engng Mater. Struct. 22 (7), 559-565.

7. Wang, Q.Y., Berard, J.Y., Dubarre, A., Baudry, G., Rathery, S. and Bathias, C. (1999). Gigacycle fatigue of ferrous alloys. Fatigue \& Fract. Engng Mater. Struct. 22 (7), 667-672.

${ }^{8}$. Ko, H.N., Kagaya, C., Itoga, H., Tokaji, K. and Nakajima, M. (2001). Effect of fine particle bombarding on fatigue strength of high strength steel. In Surface Treatment V: Computer Methods \& Experimental Measurements for Surface Treatment Effects. C.A. Brebbia, WIT Press, 313-322.

${ }^{9}$. Umezawa, O. and Nagaï, K. (1997). Subsurface crack generation in HCF for high strength alloys. ISIJ International 37 (12), 1170-1179.

${ }^{10}$. Stephens, R.I. and Fuchs, H.O. (2001). Metal Fatigue in Engineering, Wiley-Interscience.

${ }^{11}$. Murakami, Y., Kawakami, K. and Duckworth, W.E. (1991). Quantitative evaluation of effects of shape and size of artificially introduced alumina particles on the fatigue strength of 1.5Ni-Cr-Mo (En24) steel. Int. J. Fatigue 13 (6), 489-499.

${ }^{12}$. Czelusniak, A., Morawski, C.D. and Liliental, W.K. (1994). Automatic nitriding potential control in gas nitriding. In Equipment and Processes, April 18-20 1994, Schaumburg, Illinois, 449-454.

${ }^{13}$. Prevey, P.S. (1986). X-ray diffraction residual stress techniques. Materials Characterization, ASM Handbook Vol.10, 380-392.

${ }^{14}$. Van Wiggen, P.C., Rozendaal, H.C.F. and Mittemeijer, E.J. (1985). The nitriding behaviour of Fe-Cr-C alloys. J. Mater. Sci. 20, 4561-4582.

${ }^{15}$. Mencik, J. (1986). Mechanics of components with treated or coated surfaces. Solid Mechanics \& its Applications, Kluwer Academic Publishers.

16. Jones, B.K. and Martin, J.W. (1978). Fatigue failure mechanisms in a nitrided En 41B steel. Metals Technology 5 (7), 217-221.

${ }^{17}$. Murakami, Y., Nomoto, T. and Ueda, T. (1999). Factors influencing the mechanism of superlong fatigue failure in steels. Fatigue \& Fract. Engng Mater. Struct. 22 (7), 581-590.

${ }^{18}$. Limodin, N., Verreman, Y. and Tarfa, N.T. (2002). Fatigue behaviour of a nitrided 4140 steel: competition between two mechanisms. In Fatigue 2002 Vol.5, Stockholm, Sweden, June 2002, 3163-3170.

${ }^{19}$. Ebert, L.J., Krotine, F.T. and Troiano, A.R. (1966). Why case hardened components fracture. Metal Progress September 1966, 61-64 
${ }^{20}$. Jack, D.H. and Winnik, S. (1979). Effect of process variables on the fatigue resistance of nitrided steels. In Heat Treatment 79. Birmingham, England, May 22-24 1979, 169-177.

${ }^{21}$. Qian, J., Fatemi, A. and Cordes, T.S. (1993). The influence of subsurface nucleation on uniaxial fatigue behaviour of ion nitrided specimens. In Fatigue 93 Vol.1, Montreal, Canada, May 3-7 1993, 329-334.

${ }^{22}$. Verreman, Y. and Espinosa, G. (1997). Mechanically short crack growth from notches in a mild steel. Fatigue \& Fract. Engng Mater. Struct. 20 (2), 129-142.

${ }^{23}$. Kim, C., Diesburg, D.E. and Eldis, G.T. (1982). Effect of residual stresses of surface hardened steels - An analytical model. Residual Stress Effects in Fatigue ASTM STP 776, 224-234.

${ }^{24}$. Kato, M., Deng, G., Inoue, K., and Takatsu, N. (1993). Evaluation of the strength of carburized spur gear teeth based on fracture mechanics. JSME International Journal Series C 36 (2), 233-240. 
